\title{
Feedback Control of a Foldable Delta Mechanism with Integrated Inkjet-Printed Angle Sensors
}

This paper was downloaded from TechRxiv (https://www.techrxiv.org).

LICENSE

CC BY-NC-SA 4.0

SUBMISSION DATE / POSTED DATE

09-10-2021 / 18-10-2021

CITATION

Türkmen, Dila; Acer Kalafat, Merve (2021): Feedback Control of a Foldable Delta Mechanism with Integrated Inkjet-Printed Angle Sensors. TechRxiv. Preprint. https://doi.org/10.36227/techrxiv.16688929.v1

$\mathrm{DOI}$

10.36227/techrxiv.16688929.v1 


\title{
Feedback Control of a Foldable Delta Mechanism with Integrated Inkjet-Printed Angle Sensors
}

\author{
Dila Turkmen, Merve Acer Kalafat
}

\begin{abstract}
Foldable robotics is accepted as one of the leading technologies in the soft robotics field. Integrating the sensing components, including hinge angle proprioception, into the robot with a single fabrication method is a part of the field's ultimate goal. Here we present a cheap single-step method for angle sensing integration into the hinges, with an accurate and reproducible performance. We use silver nanoparticle inkjet printing on the flexible structural layer (PET) of the foldable robot (i.e. Delta robot), using an office-type printer. Silver printed sensors were studied for slight bending applications; however, we report their behavior under a $1 \mathrm{~mm}$ minimum radius of curvature, an advanced range both for silver strain sensors and any printed hinge position sensors. Among the three patterns studied, one gave a mean absolute dynamic hysteresis error below $1^{\circ}$. Reproducibility of a printed angle sensor behavior is reported for the first time, with three prototypes of each pattern $\left(2^{\circ}\right.$ standard deviation). Printed sensor feedback is tested with proportional control for the first time, via set-point and tracking tasks. Onoff control law is also implemented and errors below $1^{\circ}$ are achieved. Proportional control performances are compared with encoder feedback control and the difference between the realized trajectories are found to be under $1 \mathrm{~mm}$ in the task plane.
\end{abstract}

Index Terms-Printed sensors, angle sensors, flexible sensors, foldable robotics

\section{INTRODUCTION}

$\mathbf{F}$ OLDABLE robotics (origami folding) is one of the leading technologies in the soft robotics field. It provides a simple method for achieving complex 3D structures from flat, 2D components. The planar fabrication techniques such as photolithography, laser machining, planar printing, and pick\&place assembly are simpler comparing with the conventional lines used for rigid fixture alignments. It was theoretically proved that generating any 3D shape from a 2D flat paper is possible. Although it has some practical limitations, today, folding patterns for various $3 \mathrm{D}$ shapes can be designed by using computational tools [1]. The flat structure of

D. Turkmen and M.Acer Kalafat was with the Department of Mechanical Engineering, Flexible Systems Laboratory, Istanbul Technical University, Turkey,

e-mail: turkmend@itu.edu.tr, acerm@itu.edu.tr foldable robots also provides compact transportability, which makes them candidates for space missions [2]. Their relatively easy, fast, and cheap fabrication makes this class of soft robots advantageous both for laboratory research\&development [3], [4] and single-use products (i.e. medical and food industry) [5]-[7].

It is important to embed the sensing units in the soft robot structure with a single low-cost fabrication process and without using any hard components to achieve an all-integrated device, which is the furthest objective in the field [8]. Joint position proprioception in robots can be used for tasks such as reconstruction (e.g. exploration) [9]-[14], set-point control (e.g. reconfiguration, pick\&place, gait control) [13]-[16] or tracking control (e.g. micromanipulation) [17], [18]. Most existing foldable robots are working in open-loop. Six studies report angle sensing. Two of them employs a hard components (i.e. photoresistor, slider\&potentiometer) [15], [18]. Paik et. al. use a microfluid injection method which is a relatively complex method and yields a highly nonlinear response [19]. One other method for embeddable sensor fabrication is screen printing which requires multiple steps (e.g. mask fabrication) and limits detailed pattern printing, and so the scalability [13], [16]. Inkjet printing is a simple and low-cost fabrication method, that allows detailed patterns with print thickness under $0.5 \mu \mathrm{m}$. Sun et. al. use carbon inkjet-printed sensors for foldable robot joint angle sensing [14]. However, it requires three-layer printing, two for the carbon ink sensor and one for the electrodes\&circuitry.

In this study, we achieved to fabricate a foldable Delta robot with angle feedback in its active joints, without adding any extra assembly steps to the existing fabrication process of the sensorless Delta robot [20]. We have printed resistive sensors on the already existing structural flexible PET layer of the robot using an office type inkjet printer and silver nanoparticle (Ag NP) ink. Using the same ink both for the sensors and the electrodes eliminated the need for any multiple layer printing. Although Ag NP inkjet printed strain sensors have been studied for linear strains or bending sensors [12], [21], [22], we have studied their behavior under such a low 


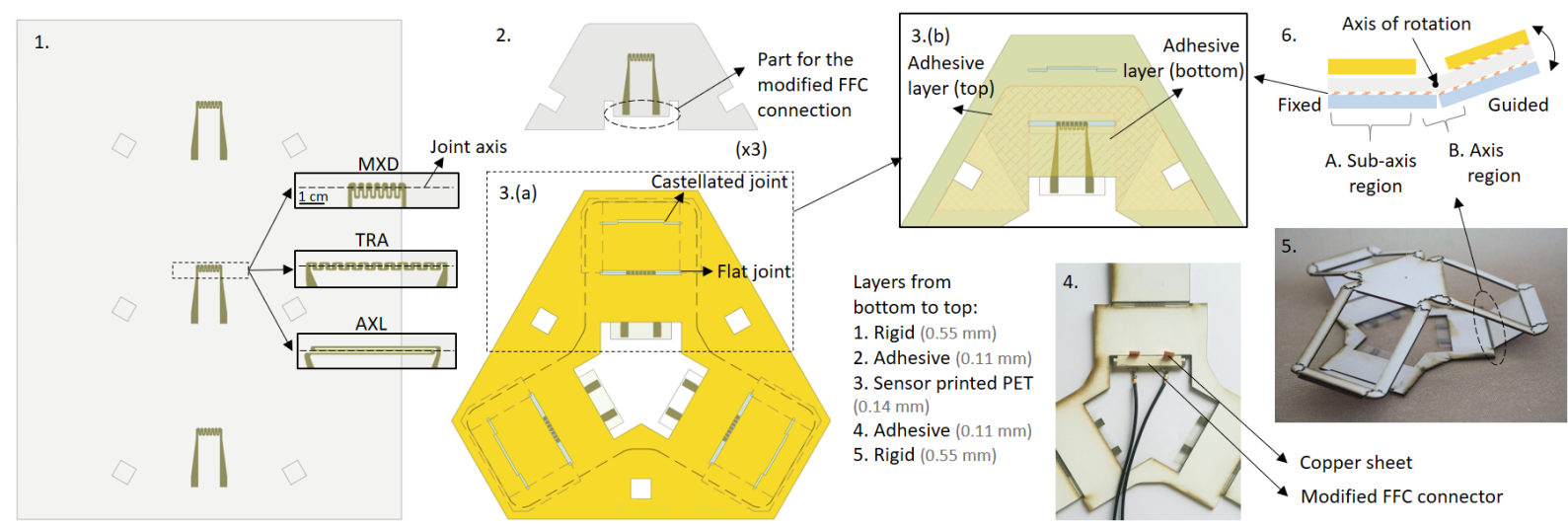

Fig. 1. Integration of the sensors into the Delta Mechanism. (1-3) show the sensor integration steps into the mechanism's base platform. Sensors are printed as in (1) and cured. Then they are cut in the laser cutting machine into pieces given in (2). (3.(a)) shows the assembled bottom platform. Layers are listed in the figure and shown in the transparent close-up (3.(b)). After the sensor integration, platform is cut through the lighter dashed lines given in (3.(a)). The straightforward fabrication methodology is followed [20] to fabricate the top platform, assembly the top and the bottom, and perform the final cut (Darker dashed line in (3.(a))) resulting with the mechanism shown in (5). Square cuts on the sides are to align the layers in the assembly step, using a 3D printed guide. (4) shows the bottom platform after the final cuts, with an assembled connector, and (5) the completed Delta mechanism. (6) shows the excitation scheme of the flexible hinge. Three different sensor patterns studied (MXD, TRA, and AXL) are shown in (1).

radius of curvature $(1 \mathrm{~mm})$ for the first time. This radius of curvature is also the lowest achieved for any soft angle sensor integrated foldable robot (Table I). Three sensor patterns are studied. One of them is found to give a mean absolute dynamic hysteresis error under $1^{\circ}$ (Table I). As the Delta mechanism contains three joints, three separate sensors were fabricated at a time, and their ramp responses are found to be reproducible $\left(2^{\circ}\right.$ standard deviation) under the same fabrication conditions. Thus, the sensor behavior reproducibility data for a printed angle sensor is reported for the first time.

Hard component DC motors are used for joint actuation to see the printed sensor effect in the feedback control. On-off control implementations with the printed sensors gave $0.5^{\circ}$ accuracy with a $0.8^{\circ}$ standard deviation. The corresponding data reported in the literature is above $1^{\circ}$, while not providing proper comparability as these studies use soft actuators with unknown contributions to the overall inaccuracy.

As a final contribution, we present proportional and tracking control implementations using a printed angle sensor feedback for the first time. Drawing star and circle shapes on the task space were realized with submillimeter level discrepancies in the task plane, when compared with the encoder feedback control case.

We present all the performance parameters in a comparison table with the literature. At the end of the paper we also state the main factors that we observe to cause errors in the sensor and control performances (e.g. acceleration dependency, cyclic drift, short lifetime), and suggest possible focuses for further studies. A further challenge in the sensor integrated foldable robots field is also stated. We believe the proposed method is promising in terms of providing a single-step fabrication process for hinge angle sensors, and we expect that the findings would contribute to the field in extending the printed sensor implementations for feedback control.

This paper is organized as follows: Section II introduces the fabrication, measurement, working principle, sensor design, and control methods. Section III presents experimental results of dynamic and static behaviours of three sensor patterns and the sensor's control performances integrated into the Delta robot. Finally, the work is concluded with discussions and future works in Section IV.

\section{Methods}

\section{A. Fabrication}

A sensorless Delta mechanism was designed and fabricated as a foldable robot, with a layer-by-layer fabrication method in our previous work [20]. In this study, we govern the same fabrication process but integrate inkjet-printed sensors into the active joints of the robot. No extra layers are added for the sensors, as the already existing single structural flexible layer is used as the printing substrate. Sensor patterns and the electrodes are printed using an Epson L382 office-type desktop printer and Novacentrix-melaton JS-B25P Ag NP inkjet ink on a $0.14 \mathrm{~mm}$ PET substrate (Novacentrix, Novellve Printing Media). As printer settings, colored print, premium glossy photo paper type, and highest print quality is selected, with the high speed option unchecked. After printing the sensors, the letter-size PET sheet is cured in Memmert UN55 oven at $120^{\circ} \mathrm{C}$ for an hour. Sensors are then cut using a laser cutting machine and 
integrated into the foldable robot structure with the layerby-layer fabrication as explained in Figure 1. Adhesive layer contact with the sensor print area is avoided, due to the damage and failures observed in the adhesive covered specimens. The remaining parts of the Delta mechanism were designed and fabricated as was presented in our previous study in detail [20], Figure 1. Note that in this study the castellated joint designs in [20] are used only in the passive joints, and flat profiles are used in the sensor integrated joints, to maintain a uniform boundary condition for the sensors (Figure 1). In case of an actuator integration other than the DC motor and the rigid setup which defines a stable joint axis for the active joints (e.g. a soft actuator), castellated joint profiles should be used for mechanical stability.

\section{B. Measurement}

For data acquisition, a simple voltage divider circuit and NI USB data acquisition card are used. An Arduino uno board is used to suply a regulated $5 \mathrm{~V}$ voltage to the circuit. Power input to the Arduino board is supplied from the PC usb port. For the sensor-circuit connection, a 30 pin FFC connector is modified. To provide the suggested thickness for the FFC and avoid deformationbased connectivity problems, a $0.15 \mathrm{~mm} \mathrm{Cu}$ sheet is inserted between the sensor and the FFC pins (Figure $1)$. With these measurement instrumentations, a $0.02 \Omega$ $(0.4 \%$ ) standard deviation is found to exist on the sensor resistance data taken with $100 \mathrm{hz}$ and for 10 seconds. The standard deviation in the $5 \mathrm{~V}$ voltage supply is measured $0.1 \%$.

Reference resistance is selected as $R_{r e f}=R_{s}$ which is the conventional approach for voltage divider design maximizing the circuit sensitivity (for neglected noises).

\section{Working Principle}

A general formula for all kinds of resistive sensors is given in Eq. 1, where $l$ is the length, $A$ is the crosssection area of the specimen, and $\rho$ is the resistivity of the material. The first two terms of the equation correspond to the geometrical effect of the strain on the sensor. Linear [23], [24] and quadratic [21] modeling approaches exist to represent this behavior. Beisteiner highlighted that the geometrical effect was not dominant for the inkjet printed strain sensors [24]. Most printed sensors are explained with the piezoresistive modeling approach in the literature, where a linear relationship is defined between the strain and the resistance change, to express the last term of Equation 1 (i.e. $\pi . E . \varepsilon, E$ : Elastic Modulus, $\epsilon$ : strain.) [23], [24]. As was mentioned by Chung, piezoresistivity is a material property based on the reversible microstructural changes and most existing sensors are not truly piezoresistive despite being malmodeled as piezoresistive [25].

$$
\frac{\Delta R}{R}=\frac{\Delta l}{l}-\frac{\Delta A}{A}+\frac{\Delta \rho}{\rho}
$$

Due to the low radius of curvature that our sensors go through $(1 \mathrm{~mm})$, strain measurement to reveal the relation between the strain input and the sensor resistance is not possible with a conventional method (i.e. on-the-shelf strain sensor placement). Instead, resistance data is collected versus the joint position data (motor encoder, $\theta$ ) and strain relation is estimated by using the theoretical $\varepsilon(\theta)$ expression. As the fixed-fixed-guided configuration requires a complex technical effort, a fixedsliding-guided configuration is used to obtain the strain relation (An external 3D printed experimental setup is used). In the latter configuration, sensor layer at the folding link side is free to slide between the sandwiching rigid layers and the strain on the material surface is given by,

$$
\varepsilon(\theta)=\frac{t \cdot d}{2} \cdot \tan \left(\frac{\theta}{2}\right)
$$

where $\mathrm{t}$ is the flexible layer thickness, $\mathrm{d}$ is the distance between the top rigid and the joint axis. $R(\varepsilon)$ is derived with,

$$
R(\varepsilon)=R\left(\varepsilon^{\prime}(\theta)\right)
$$

$\varepsilon^{\prime}(\theta)$ is the inverse of the experimentally derived strain expression.

\section{Sensor Design}

To obtain a safe lower boundary for the sensor resistance, glass transition temperature of the PET substrate $\left(120^{\circ}\right)$ and the maximum current limit $(500 \mathrm{~mA})$ of the voltage regulator (i.e. Arduino Uno) are considered. It is found that matching the latter condition was sufficient to keep the sensor below the critical temperature. This condition limits the minimum resistance of the sensors (and reference resistances) to $5 \Omega$ for the $5 \mathrm{~V}$ supply.

Sheet resistance $\left(R_{\square}\right)$ is used for the transition from desired sensor resistance to sensor geometry as below, where $r_{a}$ is the aspect ratio along the conductive path.

$$
R_{s}=R_{\square} \cdot r_{a}
$$

$R_{\square}$ was given by the manufacturer as $0.06 \Omega / \square$ for a photonic curing process [26]. With oven curing at $120^{\circ} \mathrm{C}$ for 1 hour, the minimum and maximum sheet resistances we recorded are $0.1 \Omega / \square$ and $0.2 \Omega / \square$ respectively, with an average $0.12 \Omega / \square$. Sheet resistances are obtained with number of squares method, using a straingauge pattern (396 squares). 

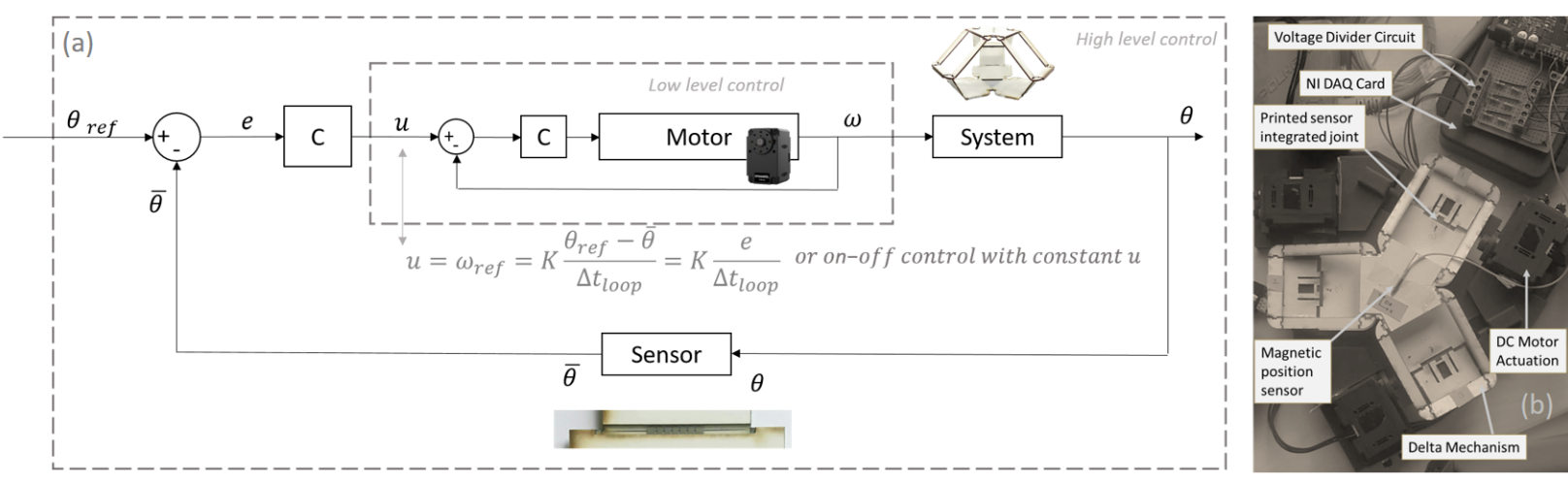

Fig. 2. (a) Block diagram for angular position control with inner velocity control loop. (b) Setup used in the characterization and control experiments.

Three different sensor patterns are designed and embedded into the Delta mechanism: AXL, with the axial current flow, TRA with the transversal current flow, and MXD with the mixed current flow, relative to the joint axis (Figure 1(1)). Serpentine patterns are used for all patterns to minimize thermal effects, although the minimum resistance limit obtained in the previous step allows flat patterns. Higher percentages of the pattern resistances are concentrated into the deformation area, to maximize the sensitivity.

For the placement of the sensor, two regions are considered: A. Sub-axis region, where the sensor does not experience any bending deformation but only a linear strain, B. Axis region, where the sensor itself is bent (Figure 1(6)). It is calculated that the strain occurring in the sub-axis region is an order below of in region B. Although region A is promising in terms of repeatability, linearity, and sensor lifetime as being a less deformative region [27], sensor responses here are estimated to be an order below of in region B, and verified to be insufficient in the preliminary studies (Supplementary Material, Section I) and region B is selected for the implementation. Adhesive free top and bottom surfaces of the active sensor region allowed free distribution of the strain. This arrangement resulted in a fixed-guided configuration for the flexible sensor layer (Figure 1(6)).

The compression and tension side printing of the sensors are also tested. Tension side is found to be more prone to failure due to the flatter thus longer crack chains observed (Supplementary Material, Section II), compared with the compression side folded sensors. The minimum width of print in the folding axis neighborhood is selected as $1 \mathrm{~mm}$ to avoid any crack-based connectivity loss.

\section{E. Control}

Dynamixel XL430 w250t servo motors are used for actuating the joints through a 3D printed setup (Figure
2 (b)). Motors are run in the velocity control mode in the low level, and the control input $u$ at the higher level is calculated using the printed angle sensor feedback, with the formulation given in Figure 2 (a). As will be introduced in the results section, some patterns yield responses with high nonlinearity and hysteresis, and some do not. Therefore, a step-wise calibration trajectory from $0^{\circ}$ to $90^{\circ}$ and back is employed to compare control performances, eliminating the calibration-based errors (Figure 4 (A.III)). For a static calibration, data is collected in the last 5 seconds of 10 seconds stop at each $10^{\circ}$ step and averaged. For a dynamic calibration, a single data is taken at each step without waiting.

Measured sensor resistance is normalized with the function in Equation 5. $R_{S}$ is the sensor resistance at the instant of the measurement, and $R_{0}$ is the sensor resistance measured in the flat position. Measured angle estimation $(\bar{\theta})$ is done with linear interpolation between the calibration points. The hysteresis effect is eliminated by using two separate data set for folding and unfolding of the joint.

$$
R_{S_{N o r m}}=\frac{R_{0}-R_{S}}{R_{0}}
$$

\section{RESULTS}

\section{A. Sensor Behaviour}

Resistance data is collected versus the joint position data (motor encoder) for a fixed-sliding-guided configuration, and found to show a cubic relationship (Figure 3). Relation between the joint angle and strain at the flexible layer surface is calculated with the formula given in Figure 3. Resulting $R(\varepsilon)$ is found to be not linear, but a $4^{\text {th }}$ order polynomial. This results indicate that the dominant mechanism shaping the sensor response is not piezoresistivity.

An other finding supporting this outcome was the major irreversible component seen in the first few cycles 


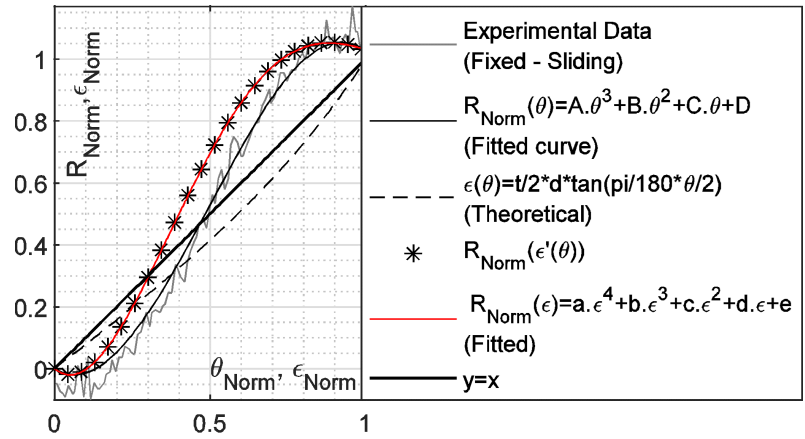

Fig. 3. Estimation of the $R(\varepsilon)$. a $=3.87, \mathrm{~b}=-10.92, \mathrm{c}=8.89, \mathrm{~d}=-0.80$, $\mathrm{e}=0, \mathrm{~A}=5 \mathrm{e}-6, \mathrm{~B}=6.6 \mathrm{e}-4, \mathrm{C}=7.1 \mathrm{e}-3, \mathrm{D}=4.5 \mathrm{e}-2$

of the sensors, and sensitivity loss seen at 300+ cycles. These observations indicate that the major effect causing the resistance change is not a reversible mechanism as piezoresistivity, but is deformative. (Cyclic response data through a sensor's life-time is provided in Supplementary Material, Section III.)

Compared with the transversal and mixed patterns, the axial patterned sensors are found to be much more reproducible under the same fabrication conditions $\left(2^{\circ}\right.$ standard deviation in the normalized folding and unfolding ramp responses of three prototypes) (Figure 4 (I)). However, it is also found to be less sensitive to joint angle change (Figure 4 (III)). TRA and AXL patterns are observed to have opposite cyclic drifts, where the MXD pattern showed no significant drift. This resulted in the MXD pattern having the highest repeatability (Figure 4. AXL patterned sensor gave the most linear response (Figure 4).

An anisotropic acceleration-dependency is recorded in all printed sensor behaviors. Sensor response for the folding motions remained unchanged, whereas the unfolding motion responses shifted with varying acceleration profile. Figure 4 (B.III) shows the shift in the unfolding response of the mixed flow sensor when the static calibration profile (stepwise) switches from sharp deceleration to smooth deceleration (i). Hysteresis disappears in the latter. For a dynamic calibration profile (i.e. triangular wave), transversal and mixed flow patterns showed hysteresis behavior (dynamic hysteresis). This behavior disappeared when a static calibration profile was used for the same sensors (Figure 4 (A.I, III \& B.I, III)). Opposingly the axial pattern showed the vice versa hysteresis behavior where no significant hysteresis is seen in the dynamic response (Figure 4 (C.I, III)). All observations are based on three prototypes of each pattern. It should be noted that, in order to indicate if these differing characteristics are arising from the patterns itself or the soft structure dependent uncertainties, further inspection must be done with a rigid setup.
Table I shows axial pattern sensor specifications compared with the existing literature of printed angle sensor integrated foldable robots. The sensor presented in this study has the advantage of using a single fabrication step and a single material as a silver inkjet printed layer. It has the lowest reported radius of curvature $(1 \mathrm{~mm})$ as an flexible angle sensor. Standard deviation in response data belonging to 3 separate sensors is $2^{\circ}$. No reproducibility performance was reported for the remaining studies, in terms of angle estimation accuracy. Using the same cubic function for all 3 prototypes (Figure 4 (C.I)), a $1.1^{\circ}$ mean absolute error is calculated for the angle estimation (Supplementary Material, Section III), Despite the nonlinear response, this error is lower than observed in [14] for a single sensor. Latter is the only printed angle sensor in the literature which is reported to have a linear response.

Maximum dynamic hysteresis seen in the ramp response is below $2.5^{\circ}$ with a mean absolute error $0.9^{\circ}$ (Figure 4(C.I)). Repeatability analysis using the data in Figure 4 (C.II) gave a $5^{\circ}$ mean error for 10 cycles. This error increase at the angular positions above $70^{\circ}$. Figure 4 (C.I) the shift between the two successive stepwise trajectory response (10 set points from $0^{\circ}$ to $90^{\circ}$ ) is shown to be below $1.4^{\circ}$. Mean set point repeatability is $0.9^{\circ}$. Step response of sensors had $0.1 \mathrm{~s}$ rise time and a static drift between $0^{\circ}$ and $2^{\circ}$ (Figure 4(B.III.ii, C.III.i)).

One general problem for all the printed angular sensors in the literature is the cyclic drift, which also exists in the axial pattern sensor $\left(10^{\circ}\right.$ for $\left.\mathrm{N}=10\right)$. One improvement on this issue was reported by Firouzeh et. al. by forming cuts on the flexible sensor layer before conductive ink deposition. However, this procedure was applied within the screen printing process. Note that the mixed pattern sensors reported in this paper showed no significant cyclic drift (Figure 4(B.II)).

\section{B. Control performances}

Characterization studies showed that the AXL pattern is more consistent in the dynamical state, and TRA and MXD patterns are in the static state. Due to the higher nonlinearity and lower sensitivity of TRA at lower angles, this pattern is not employed in the control implementations. A set-point task is performed with MXD patterned sensor feedback, consisting of 10 successive references between $50^{\circ}$ and $70^{\circ}$, with 10 seconds stops at each (Figure 5(a)). $0.5^{\circ}$ mean steadystate error and $0.8^{\circ}$ standard deviation is achieved with an $8 \% / s$ constant control input. These results show higher accuracy compared with the existing literature (Table I). It should be noted that reported studies in Table I were using soft actuators. Thus the actuator effect on the reported inaccuracies is unknown. 

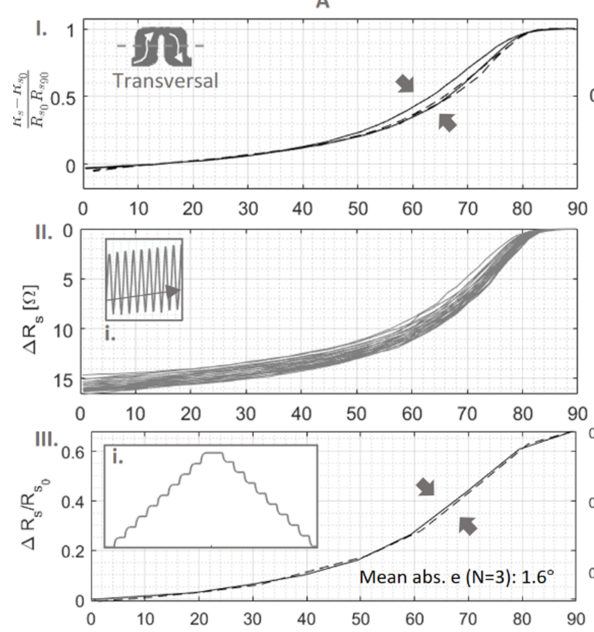
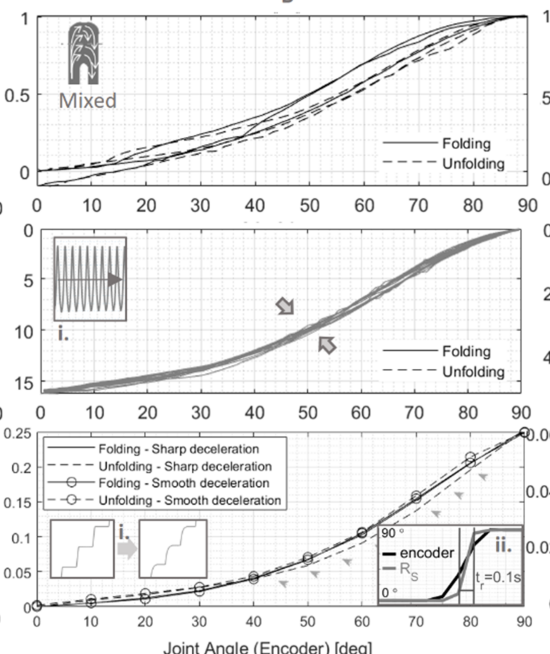
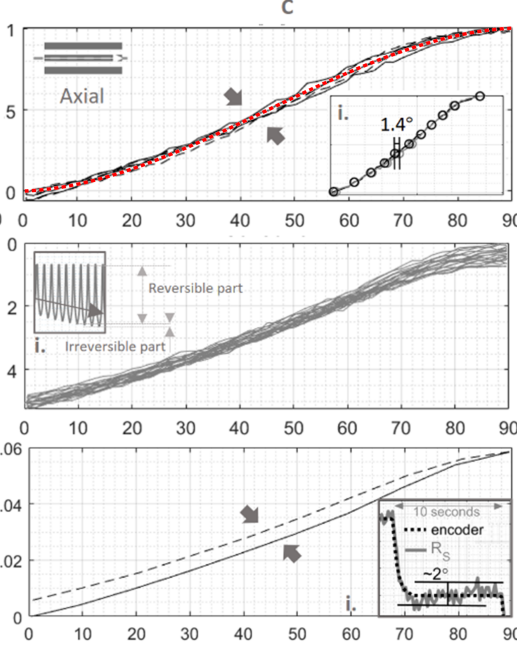

Fig. 4. Dynamic and static behaviours of three distinct sensor patterns; TRA (coulum A), MXD (coulum B) and AXL (coulum C). Row I. presents folding and unfolding behaviours of the sensors for three prototypes of each pattern (ramp responses). In C.I.i, dynamic hysteresis of a single AXL prototype is presented for clearity. Row II. shows the shifts in the sensor response for 10 repetitive triangular input cycle, for a single prototype of each pattern. Row III. represents the static response of the sensors for $10^{\circ}$ step inputs(i.), calculated by averaging the last $5 \mathrm{sec}$ data of each $10 \mathrm{sec}$ step. Graphs present a single prototype for each pattern. Mean absolute hysteresis error for three prototypes of TRA pattern is $1.6^{\circ}$. In B.III.i, smooth and sharp static calibration profiles are given, together with their effect on the unfolding response in the main graph (gray arrows). B.ii and C.i shows the step response of the printed sensors in corresponding patterns.
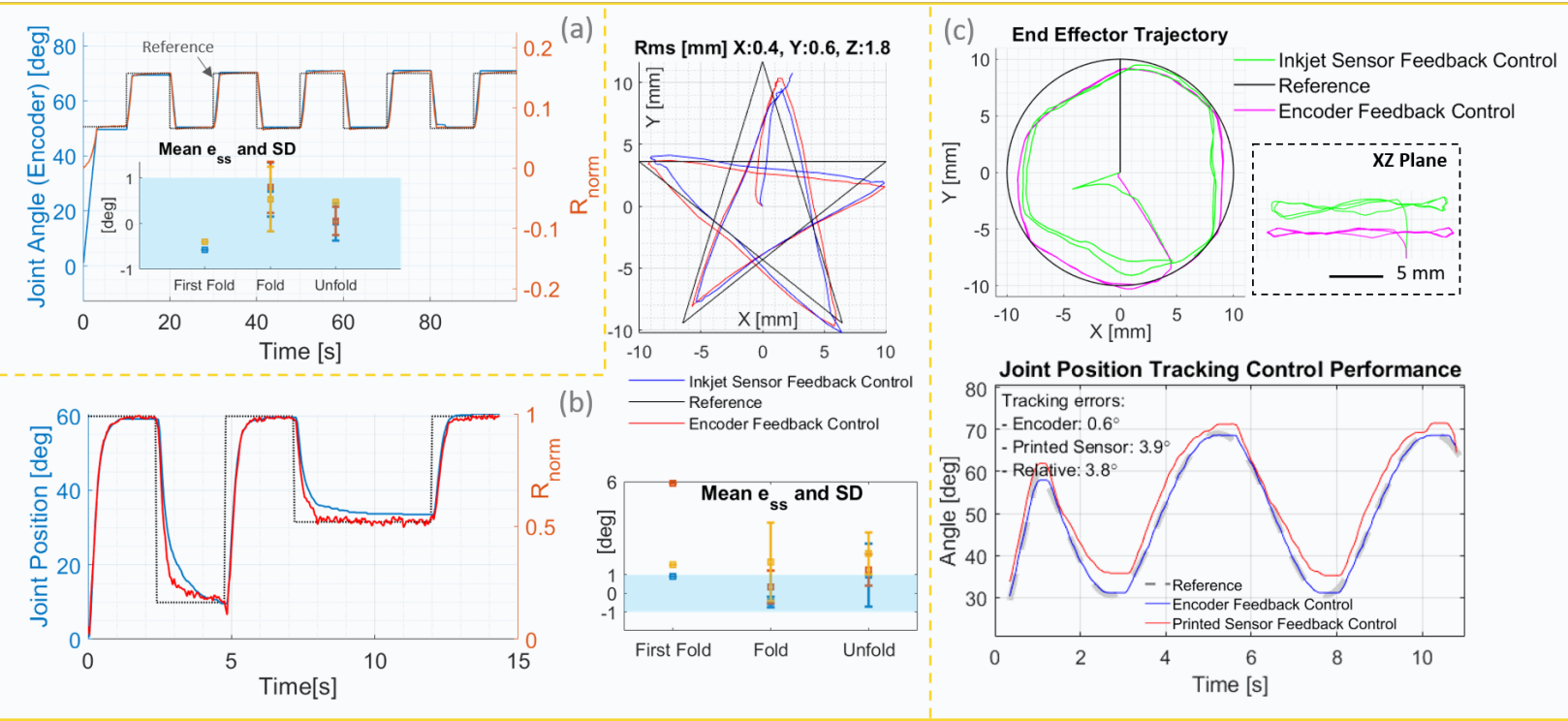

Fig. 5. Control results achieved with the printed sensors, joint and task space graphs. (For the joint space, only a single joint's results are represented out of three actuated joints of the Delta Robot. Remaining data is provided in the supplementary material.) (a) On-off control performance. (b) Set-point control performance with proportional control. Each corner of the star shape is given as set-points. (c) Tracking control performance with proportional control.

Proportional control is needed for achieving a task space reference with a synchronized motion in joint space. A star shape is drawn as a set-point control task by defining the five corners as the set-points (Figure 5(b)). In a second scenario, drawing a $10 \mathrm{~mm}$ diameter circle is realized as a tracking control task (Figure 5(c)).
For both implementations, the AXL pattern is used with dynamic calibration. One ramp cycle applied before both the calibration and the control steps is found to decrease the tracking errors by eliminating the transient effects. Both proportional control coefficients $\left(K_{p}\right)$ are tuned experimentally (set point: 0.04 , tracking 0.1 ). Notice 
TABLE I

AXIAL PATTERNED SENSOR SPECIFICATIONS COMPARED WITH THE EXISTING LITERATURE OF PRINTED ANGLE SENSOR INTEGRATED FOLDABLE ROBOTS. IN THE SECOND SECTION OF THE TABLE, SET POINT AND TRACKING CONTROL PERFORMANCES ARE PRESENTED FOR ON-OFF AND PROPORTIONAL CONTROL LAWS.

\begin{tabular}{|c|c|c|c|c|}
\hline & This Paper & Firouzeh et.al. [16] & Sun et.al. [14] & Kwak et.al. [13] \\
\hline \multicolumn{5}{|c|}{ Characterization } \\
\hline \# of Fab. Steps & Single & Multi & Multi & Multi \\
\hline Rad. of Curv. & $1 \mathrm{~mm}$ & $1.4 \mathrm{~mm}$ & NR & NR \\
\hline Fab. Method. & Inkjet & Screen Print & Inkjet & Screen P. \\
\hline Material & Silver & Carbon & Carbon & Conductive Polyme \\
\hline Actuator & Hard (DC motor) & Soft (SMA) & Soft (SMA) & - (no actuation) \\
\hline Range & $0^{\circ}-90^{\circ}$ & $0^{\circ}-180^{\circ}$ & $0^{\circ}-60^{\circ}$ & $0^{\circ}-90^{\circ}$ \\
\hline Reproducibility & $\sigma=2^{\circ}$ & NR & NR & NR \\
\hline $\begin{array}{l}\text { Nonlinearity } \\
\text { (Calib. Acc.) }\end{array}$ & $\begin{array}{c}\text { Linear Fit: }<8^{\circ} \\
\text { Cubic Fit: }<4^{\circ},|\bar{e}|=1.1^{\circ}\end{array}$ & NR & $\begin{array}{l}\text { Linear Fit*: } \\
0^{\circ}<e<15^{\circ}\end{array}$ & $\begin{array}{c}\text { (2 calib. lines) } \\
3.3^{\circ} \mathrm{RMS}\end{array}$ \\
\hline $\begin{array}{l}\text { Dynamic } \\
\text { Hysteresis }\end{array}$ & $\begin{array}{c}<2.5^{\circ} \\
|\bar{e}|=0.9^{\circ}\end{array}$ & $\begin{array}{c}\mathrm{NR} \\
\left(\text { static hys. }<10^{\circ} *\right)\end{array}$ & $\begin{array}{c}\mathrm{NR} \\
\text { (mono directional) }\end{array}$ & $<2.5^{\circ} *$ \\
\hline $\begin{array}{l}\text { Repeatability } \\
\text { (Cyclic Resp.) }\end{array}$ & $\begin{array}{l}5^{\circ}(\mathrm{N}=10) \\
\left(@ 0-70^{\circ}\right)\end{array}$ & NR & $\begin{array}{c}<9^{\circ} *(\mathrm{~N}=4) \\
\left(@ 15-45^{\circ}\right)\end{array}$ & NR \\
\hline $\begin{array}{l}\text { Repeatability } \\
\text { (Step Resp.) }\end{array}$ & $\begin{array}{l}|\bar{e}|=0.9^{\circ}(\mathrm{N}=2) \\
\quad(10 \text { set points })\end{array}$ & $\begin{array}{l}1.1^{\circ} *(\mathrm{~N}=50) \\
(20 \text { set points })\end{array}$ & $\begin{array}{l}2^{\circ}(\mathrm{N}=30) \\
(1 \text { set point })\end{array}$ & NR \\
\hline Rise Time & $0.1 \mathrm{~s}$ & NR & $0.14 \mathrm{~s}^{*}$ & \\
\hline St. Drift & $<2^{\circ}$ & NR (modelled) & $2^{\circ}$ & NR \\
\hline Cyclic Drift & $\begin{array}{l}\text { Regular Drift } \\
10^{\circ}(\mathrm{N}=10)\end{array}$ & $\begin{array}{c}\text { Regular Drift } \\
\text { NR }\end{array}$ & NR & $\begin{array}{l}\text { Irregular Drift } \\
<3^{\circ *}(\mathrm{~N}=10)\end{array}$ \\
\hline Life Time & $\sim 300$ cycles & $400+$ cycles $*$ & NR & NR \\
\hline \multicolumn{5}{|c|}{ Control Performances } \\
\hline $\begin{array}{l}\text { Set Point } \\
\text { On-Off }(\mathrm{N}=5)\end{array}$ & $\begin{array}{l}e_{s s}=0.5^{\circ} \\
S D: 0.8^{\circ}\end{array}$ & $\begin{array}{c}e_{s S} \mathrm{NR} \\
S D: 1.6^{\circ}\end{array}$ & $\begin{array}{c}e_{S S}=1.3^{\circ} \\
S D: 1.5^{\circ}\end{array}$ & (No Actuation) \\
\hline $\begin{array}{l}\text { Set Point } \\
\text { Proportional } \\
(\mathrm{N}=6)\end{array}$ & $\begin{array}{c}e_{s s}=1.8^{\circ}, S D: 0.6^{\circ} \\
\mathrm{x}: 0.4 \mathrm{y}: 0.6 \mathrm{z}: 1.8 \\
(\mathrm{~mm} \text { RMS })\end{array}$ & - & - & (No Actuation) \\
\hline $\begin{array}{l}\text { Tracking } \\
\text { Proportional }\end{array}$ & $\begin{array}{c}3.8^{\circ} \text { RMS } \\
\mathrm{x}: 0.7 \mathrm{y}: 1.1 \mathrm{z}: 3.8 \\
(\mathrm{~mm} \text { RMS })\end{array}$ & - & - & (No Actuation) \\
\hline
\end{tabular}

NR Not reported in the corresponding references.

* Not reported explicitly in the corresponding references, estimated numerical values are given based on the reported graphs.

$|\bar{e}|$ : Mean absolute error, $\sigma$ : Standard deviation.

Set-point control data: MXD pattern, tracking control data: AXL pattern.

Characterization parameters respectively: number of fabrication steps, minimum radius of curvature, fabrication method, ink material, joint actuation, sensor range, reproducibility of normalized ramp response (fold \& unfold), nonlinearity (in terms of linear calibration accuracy), dynamic hysteresis, dynamic and static repeatability based on the cyclic and step responses, rise time, static drift, cyclic drift, life time. 
the low controller gain for the set-point control task decreases the response speed but provides a smoother trajectory for the joint thus keeping the AXL sensor in the dynamic state (A preliminary experiment where the MXD pattern is used for the star drawing task is also provided as supplementary material).

For both task space operations, relative errors in the XY planar motion stayed around $1 \mathrm{~mm}$ (Table I, Figure $5(b, c))$. Due to the lower accuracy seen in the unfolding side motions for both tasks, an off-set error is observed at Z-axis. Mean errors increased compared with the onoff control results, arising from the same inaccuracy. This behavior in the unfolding direction matches with the anisotropic acceleration dependency reported in the previous section. Unlike the proportional control, it is possible to eliminate this effect in the on-off control operation due to the constant-velocity control inputs. The distorted circle shape obtained with both sensors feedback is due to the assembly errors of the Delta mechanism and lag between the control inputs commanding the three motors. Performance metrics are reported in Table I and Figure 5. A representative single sensor data is given for each experiment, and the remaining data is provided in the supplementary material.

\section{CONCLUSION AND Discussions}

In this study an all-Ag $\mathrm{Np}$ printed angle sensor is realized for the first time. Fabricated sensors in different patterns are found to give different characteristics in terms of linearity, sensitivity, dynamic and static hysteresis behavior and cyclic drift. Regarding the uncertainties introduced by the soft structure (paper layers) and layerby-layer fabrication method, we suggest a systematical characterization analysis of the sensors using a rigid setup would be sufficient to reveal the pattern and other parameters effect (e.g. center of rotation, minimum radius of curvature) on the sensor behaviour.

Our experiments showed that it is possible to achieve a reproducible angle sensor, with calibration error around $\sim 1^{\circ}$, using the proposed method. This performance together with the hysteresis, repeatability and step response metrics, places the sensors in a competitive position within the existing literature. A detailed performance comparison is presented as a table. Sensors have the significant advantage of requiring only a single fabrication step, and a single layer printing with a single ink.

Theoretically, angle sensors operated down to a $1 \mathrm{~mm}$ radius of curvature, defined by the top rigid layer (paper) distances. This was the lowest radius of curvature realized for a flexible angle sensor. However in practice due to the fabrication uncertainties and deformable structure of the papers used as the rigid layers, it is likely that the radius of curvature at $90^{\circ}$ is slightly higher than 1 $\mathrm{mm}$. At this operating range sensors are found to have a
300 cycles life-time, before getting insensitive at the low angles due to over-deformation. However we assume the life-time of the sensors can be extended by decreasing the total width of the patterns which would limit the sensors to a less deformative region, away from the the flexible layer side edges.

Printed sensor feedback had successfully provided an accuracy below $1^{\circ}$ with on-off control. This was a higher performance than other reported hinge angle sensors'. However to correctly compare the sensor performances with the literature, the actuators should also match, which is not true in this case.

We presented the first implementation of printed angle sensors with proportional control law and tracking control task. Main difference of the proportional control from the on-off control which was employed in the remaining literature, is the control signal being dynamic (i.e. non-zero acceleration). The major cause of the control errors are observed to be the acceleration dependency seen in the sensor responses. This character was not studied in the remaining literature. We believe this metric should be studied further, for a wider implementation of printed sensors in feedback control.

We note that sensors were calibrated using two separate calibration data set for fold and unfold to eliminated hysteresis effect on the observations. Also the nonlinearity errors are eliminated by using 10 data points for each direction. However one of the fabricated patterns (AXL) showed a reproducible cubic response without significant hysteresis. A three point calibration, or a two point calibration by assuming a constant slope at the deflection point is possible which would be more feasible for control implementations.

One other drawback of the printed sensors are observed to be the cyclic drift, as was reported also by the other studies in the literature. One of three patterns tested in this study yielded no significant cyclic drift. However other two patterns experienced large cyclic drifts in opposite directions. This indicates a detailed characterization study may enable an optimized design without a significant drift.

Despite the latter two imperfections, when compared with an encoder feedback control case, printed sensor feedback control errors stayed approximately below $1^{\circ}$ in task plane. Errors normal to the task plane were recorded higher due to the mechanism kinematics.

Noting that the presented mechanism (i.e. Delta) is in the class of parallel-foldable-robots, a further challenge in the field can be addressed as designing printed sensors for serial-foldable-robots or serial unactuated joints, where the conductive pathways of the sensors are likely to be subjected to multiple joint deformations. 


\section{ACKNOWLEDGMENT}

This work was supported by the Scientific and Technological Research Council of Turkey (TUBITAK) (Grant No. 216M193).

\section{REFERENCES}

[1] D. Rus and M. T. Tolley, "Design, fabrication and control of origami robots," Nature Reviews Materials, vol. 3, no. 6, pp. 101-112, 2018.

[2] "A-puffer, a foldable robot that can access tight spaces," https://www.jpl.nasa.gov/robotics-at-jpl/a-puffer, accessed: 202107-06.

[3] C. Majidi, "Soft robotics: a perspective-current trends and prospects for the future," Soft robotics, vol. 1, no. 1, pp. 5-11, 2014.

[4] N. Bezzo, A. Mehta, C. D. Onal, and M. T. Tolley, "Robot makers: The future of digital rapid design and fabrication of robots," IEEE Robotics Automation Magazine, vol. 22, no. 4, pp. 27-36, 2015

[5] D. Mohanta, S. Mohapatra, S. Khuntia, S. Sahoo, and P. R. Rauta, "Origami-engineered structures and their biomedical applications," in Nanotechnology in Biology and Medicine. CRC Press, 2019, pp. 167-173.

[6] E. Vander Hoff, D. Jeong, and K. Lee, "Origamibot-i: A threadactuated origami robot for manipulation and locomotion," in 2014 IEEE/RSJ International Conference on Intelligent Robots and Systems, 2014, pp. 1421-1426.

[7] M. Johnson, Y. Chen, S. Hovet, S. Xu, B. Wood, H. Ren, J. Tokuda, and Z. T. H. Tse, "Fabricating biomedical origami: a state-of-the-art review," International journal of computer assisted radiology and surgery, vol. 12, no. 11, pp. 2023-2032, 2017.

[8] "The soft touch of robots," Nature Reviews Materials, vol. 3, p. 71, 2018.

[9] T. L. Mohren, T. L. Daniel, S. L. Brunton, and B. W. Brunton, "Neural-inspired sensors enable sparse, efficient classification of spatiotemporal data," Proceedings of the National Academy of Sciences, vol. 115, no. 42, pp. 10564-10569, 2018. [Online]. Available: https://www.pnas.org/content/115/42/10564

[10] H.-S. Shin, L. Castano, J. Humbert, and S. Bergbreiter, "Sensing skin for detecting wing deformation with embedded soft strain sensors," 2016 IEEE SENSORS, pp. 1-3, 2016.

[11] J. Tapia, E. Knoop, M. Mutný, M. A. Otaduy, and M. Bächer, "Makesense: Automated sensor design for proprioceptive soft robots," Soft Robotics, vol. 7, no. 3, pp. 332-345, 2020, pMID: 31891526. [Online]. Available: https://doi.org/10.1089/soro.2018.0162

[12] S. Zhang, L. Cai, W. Li, J. Miao, T. Wang, J. Yeom, N. Sepúlveda, and C. Wang, "Fully printed silver-nanoparticle-based strain gauges with record high sensitivity," Advanced Electronic Materials, vol. 3, no. 7, p. 1700067, 2017.

[13] B. Kwak and J. Bae, "Compliant mechanosensory composite (cmc): a compliant mechanism with an embedded sensing ability based on electric contact resistance," Smart Materials and Structures, vol. 27, no. 12, p. 125003, 2018.

[14] X. Sun, S. M. Felton, R. J. Wood, and S. Kim, "Printing angle sensors for foldable robots," 2015 IEEE/RSJ International Conference on Intelligent Robots and Systems (IROS), 2015.

[15] M. E. Nisser, S. M. Felton, M. T. Tolley, M. Rubenstein, and R. J. Wood, "Feedback-controlled self-folding of autonomous robot collectives," in 2016 IEEE/RSJ International Conference on Intelligent Robots and Systems (IROS). IEEE, 2016, pp. 1254-1261.

[16] A. Firouzeh, A. F. Amon-Junior, and J. Paik, "Soft piezoresistive sensor model and characterization with varying design parameters," Sensors and Actuators A: Physical, vol. 233, pp. 158-168, 2015 .
[17] M. Luo, E. H. Skorina, W. Tao, F. Chen, S. Ozel, Y. Sun, and C. D. Onal, "Toward modular soft robotics: Proprioceptive curvature sensing and sliding-mode control of soft bidirectional bending modules," Soft robotics, vol. 4, no. 2, pp. 117-125, 2017.

[18] M. Mete and J. Paik, "Closed-loop position control of a selfsensing 3-dof origami module with pneumatic actuators," IEEE Robotics and Automation Letters, vol. 6, no. 4, pp. 8213-8220, 2021.

[19] J. K. Paik, R. K. Kramer, and R. J. Wood, "Stretchable circuits and sensors for robotic origami," in 2011 IEEE/RSJ international conference on intelligent robots and systems. IEEE, 2011, pp. 414-420.

[20] M. Acer Kalafat, H. Sevinc, S. Samankan, A. Altinkaynak, and Z. Temel, "A novel origami inspired delta mechanism with flat parallelogram joints," Journal of Mechanisms and Robotics, pp. $1-22,2020$.

[21] S. Kim, S. Won, G.-D. Sim, I. Park, and S.-B. Lee, "Tensile characteristics of metal nanoparticle films on flexible polymer substrates for printed electronics applications," Nanotechnology, vol. 24, no. 8, p. 085701, 2013.

[22] B. Ando and S. Baglio, "All-inkjet printed strain sensors," IEEE Sensors Journal, vol. 13, no. 12, pp. 4874-4879, 2013.

[23] W.-T. Park, Piezoresistivity. Dordrecht: Springer Netherlands, 2012, pp. 2111-2117.

[24] C. Beisteiner and B. G. Zagar, "A survey of inkjet-printed lowcost sensors," tm-Technisches Messen, vol. 85, no. 7-8, pp. 504514, 2018.

[25] D. Chung, "A critical review of piezoresistivity and its application in electrical-resistance-based strain sensing," Journal of Materials Science, pp. 1-30, 2020.

[26] Melaton JS-B15P Melaton JS-B25P Melaton JS-B35P Nanosilver Ink - Aqueous dispersion, Novacentrix, 92011.

[27] C. T. Gentile, M. Wallace, T. D. Avalon, S. Goodman, R. Fuller, and T. Hall, "Angular displacement sensors," Feb 1992.

Dila Türkmen is a Ph.D. student and Research Assistant in Istanbul Technical University, Mechanical Engineering Department, where she completed her B.S. and and M.S. degrees in 2013 and 2016 resp.

Dr. Merve Acer Kalafat received her B.S. degree in Mechanical Engineering from Istanbul Technical University (ITU), Turkey, in 2005. She completed her M.S and Ph.D. in Mechatronics Engineering in Sabanci University (SU), Turkey, in 2007 and 2012. 2005 to 2012, she has worked as a Research Assistant at SU, Microsystems Laboratory. She has been an instructor in the Mechanical Engineering department at ITU in 2012. During 2013-2014 she has worked as a post-doc researcher in École Polytechnique Fédérale de Lausanne at Reconfigurable Robotics Laboratory. She established Flexible Systems Laboratory under Mechanical Engineering Department in 2018 at ITU. Her fields of interest are soft robotics, soft sensors, compliant mechanisms, origami-based structures, mechatronics, and motion control. 